Proc. Indian Acad. Sci. (Earth Planet. Sci.), Vol. 95, No. 2, July 1986, pp. 193-200.

(C) Printed in India.

\title{
Absorption of radio waves during a solar eclipse
}

\author{
D B PATEL, K M KOTADIA, P D LELE and K G JANI \\ Physics Department, Gujarat University, Ahmedabad 380009, India \\ MS received 12 April 1985; revised 16 January 1986
}

\begin{abstract}
In this paper, the results of our observations on Al-method ionospheric absorption of radio waves on 1.8 and $2.2 \mathrm{MHz}$ during the solar eclipse of 16 February 1980 are presented. The absorption decreased by about $41 \%$ and $46 \%$ of the normal value respectively at the above two frequencies at Ahmedabad following the maximum phase of the eclipse (about $77 \%$ of full disc) with a delay of 18 minutes. The quantity $A_{T}(f)$ which is a measure of $\int N v \mathrm{~d} h$ is now examined for better clarity of the influence of the changes in the $E$-layer. The results are discussed in relation to the observations of the ionizing radiations from the sun, changes in the electron density, recombination rate and absorption in the underlying $D$ and $E$ regions.
\end{abstract}

Keywords. Ionospheric radio wave absorption; solar eclipse.

\section{Introduction}

A rare event of total solar eclipse of about $150 \mathrm{sec}$ duration occurred on 16 February 1980 and the belt of totality, about $120 \mathrm{~km}$ wide, crossed the plateau of South India along the path from Karwar $\left(14.6^{\circ} \mathrm{N}, 74^{\circ} \mathrm{E}\right)$ to Puri $\left(20^{\circ} \mathrm{N}, 86^{\circ} \mathrm{E}\right)$ during the interval 1008-1020 hr U.T. At Ahmedabad $\left(23^{\circ} \mathrm{N}, 72.6^{\circ} \mathrm{E}\right)$, the maximum eclipse was $77 \%$. Figure 1 shows the map of India depicting the maximum eclipse and the time of its

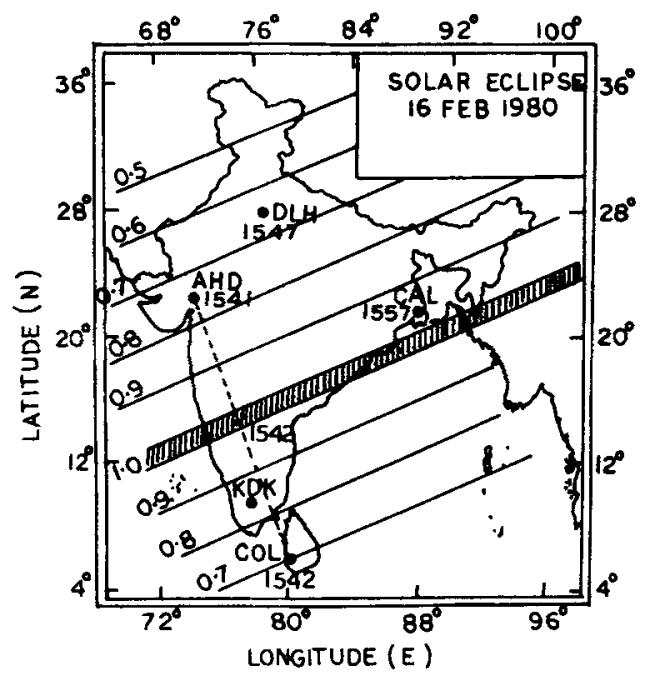

Figure 1. Map of India and Sri Lanka showing maximum percentage eclipse, contour-lines and the time of the maximum phase of solar eclipse at some selected places. AHD: Ahmedabad, DLH: Delhi, CAL: Calcutta, KDK: Kodaikanal, COL: Colombo and at midpoint of Ahmedabad-Colombo path in the totality zone. 
occurrence in IST (U.T. $+5.5 \mathrm{hr}$ ). We were particularly interested in observing the changes in ionosphere which affect radio communication in SW band. Such observations have been made and interpreted in several ways earlier (Rawer 1956; Piggott 1956; Davies 1956; Landini et al 1966; Bischoff and Taubenheim 1967; Sechrist 1970). Some interesting results on ground-based and rocket-borne observations were reported for the annular solar eclipse of 20 May 1966 at a mid-latitude place in Greece. A preliminary report on some results of ionospheric radio effects at Ahmedabad during the eclipse of 16 February 1980 was published earlier (Jani et al 1982). Here, we present results of further studies made on the changes of ionospheric absorption on 1.8 and $2.2 \mathrm{MHz}$ that took place during this solar eclipse.

\section{Total radio pulse absorption and $\boldsymbol{A}_{T}(f)$}

Al-absorption $(L, \mathrm{~dB})$ of radio pulses was measured at intervals of $15 \mathrm{~min}$ on the day of eclipse and 30 minutes on \pm 3 control days. The mean values on control days were almost the same as monthly median values at corresponding hours and were therefore taken as normal values.

As reported earlier (Jani et al 1982) the total absorption on the normal day and that on the eclipse day at the time corresponding to the lowest value of absorption 18 minutes after the maximum phase was as follows:

$$
\begin{aligned}
& L_{t N}(1 \cdot 8)=32 \mathrm{~dB} ; L_{t E}(1 \cdot 8)=19.0 \mathrm{~dB} ; \text { decrease }=41 \% \\
& L_{t N}(2 \cdot 2)=23 \mathrm{~dB} ; L_{t E}(2 \cdot 2)=12.5 \mathrm{~dB} ; \text { decrease }=46 \%
\end{aligned}
$$

Subscripts $t, N$ and $E$ stand for total, normal and eclipse respectively. The fall in absorption began with the commencement of the eclipse, initially at a slower rate and then at a faster rate after the eclipse reached $40 \%$ (i.e. $60 \%$ unobscured disc). During the time interval of the eclipse both on the control days and on the eclipse-day, there was no low-type blanketing $E s$ which produce a decrease in total absorption by an amount nearly equal to the deviative absorption in the E-layer (Kotadia et al 1977).

Since the total absorption $\left(L_{t}\right)$ is a mixture of non-deviative absorption $\left(L_{n d}\right)$ especially in the $D$-region and deviative absorption $\left(L_{d}\right)$ in the $E$-region which varies in a complex manner, it is appropriate for interpretation of results to follow the method proposed by George (1971), and Samuel and Bradley (1975) which gives a quantity called $A_{T}(f)$ expressed in $\mathrm{dB} . \mathrm{MHz}^{2}$. This takes into account a time-dependent function $\phi_{n}\left(f_{r} / f \circ E\right)$ where $f_{r}$ is the observing radio frequency, and $f_{0} E$ is the ordinary-wave critical frequency of the E-layer. In the eclipse function (1-f) $\cos \chi$ for the eclipse-day, $\chi$ is the solar zenith angle (SZA) and $f$ denotes fraction of the sun's disc eclipsed. Since both (1- $f$ ), i.e. visible part of the disc and $\cos \chi$ decrease upto the time of maximum phase, and later change in reverse order, the slope of the $(1-f) \cos \chi$ curve is steeper before the maximum phase than that after it. As a result of such dissimilarity, the electron density $N(h)$-profile and the height of reflection undergo changes in such a way, as explained by Leid (1956), that the total of two components $L_{n d}$ and $L_{d}$ varies in a complicated manner. The study of $A_{T}(f)$ which is a good measure of $\int N v \mathrm{~d} h, v$ being the collision frequency, greatly reduces the confusion arising from such a situation.

The values of $A_{T}(1 \cdot 8)$ and $A_{T}(2 \cdot 2)$ calculated for the eclipse-day and control days at corresponding times are shown in figure 2 along with the eclipse-SZA function. It may be 


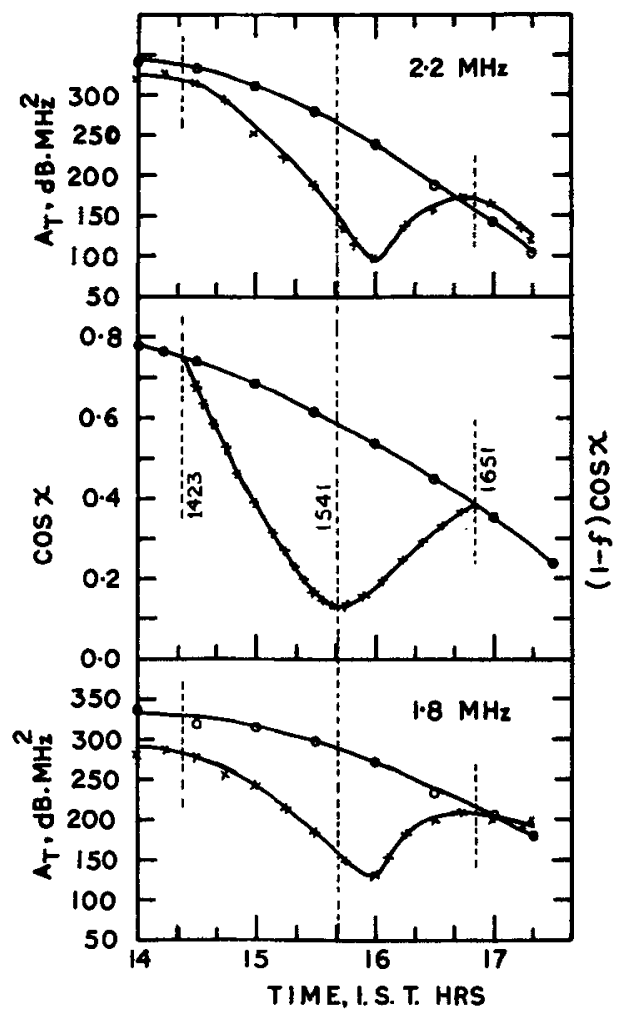

Figure 2. Variation of $A_{T}(1.8)$ and $A_{T}(2 \cdot 2)$ during the solar eclipse of 16 February 1980 compared with its control days' mean value. Also shown are the time variation of $\cos \chi$ and eclipse-associated $(1-f) \cos \chi$. Times of first contact, maximum phase and last contact of the eclipse are marked by vertical dashed lines.

stated that the points for $A_{T}(f)$ lie on a smooth curve as compared to the scatter that was seen in total absorption reported earlier. But still, the delay time of about $18 \mathrm{~min}$ and the asymmetry between the variation of $A_{T}(f)$ before and after the maximum eclipse remain. Almost the same delay was found in the maximum drop of $f \circ E$ on the eclipse-day from the normal value. It fell from $3.5 \mathrm{MHz}$ to $2.7 \mathrm{MHz}$, i.e. the $E$-layer maximum electron density $\mathrm{NmE}$ decreased by $45 \%$ accompanied by a rise in height $\mathrm{hmE}$ from $110 \mathrm{~km}$ to $120 \mathrm{~km}$ (Chandra et al 1981).

\section{Unobscured solar disc and change in $A_{T}(f)$}

In order to see the effect of the eclipse clearly as to how much was the reduction in $A_{T}(f)$ and in what manner it varied, the ratio $A_{T E} / A_{T N}$ is plotted against time in figure 3. Also shown is the unobscured part (1-f) of the solar disc, $\cos \chi$ being a common factor on control days and the eclipse-day. It may be seen that the fall in $A_{T}(f)$ in the beginning was small, but became rapid after the optically visible part of the disc fell below $60 \%$. Values of the fall in $A_{T}(f)$ corresponding to the fraction of the solar disc left unobscured are given in table 1 . Thus for a decrease in the visible part of the sun's 


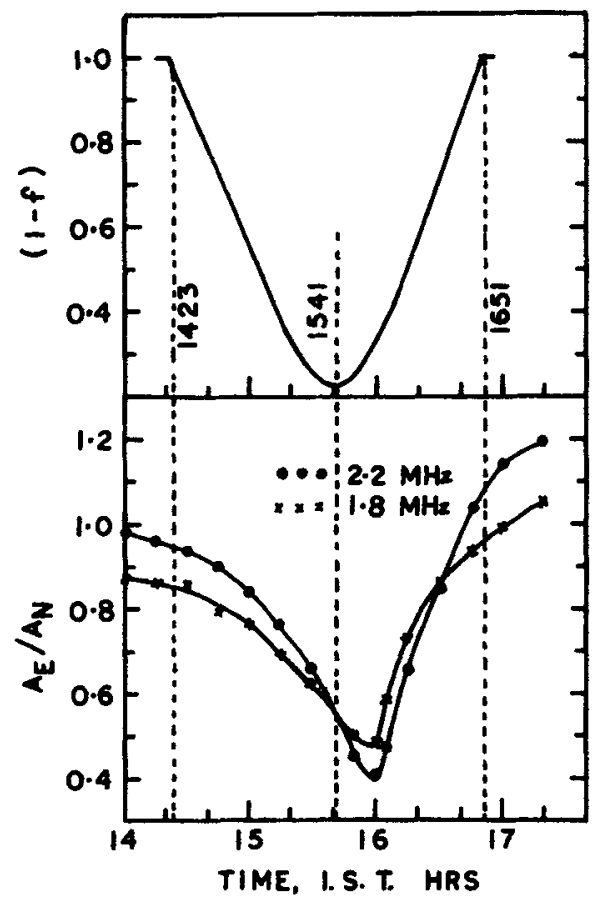

Figure 3. Variation of ratio $A_{T E} / A_{T N}$ on eclipse-day on 1.8 and $2.2 \mathrm{MHz}$. The fraction of the solar disc not eclipsed is also shown for comparison.

Talle 1. Decrease in $A_{T}(f)$ at instants of different visible parts of the solar disc relative to normal value for fully visible disc.

\begin{tabular}{ccccc}
\hline & \multicolumn{2}{c}{$A_{T}(1 \cdot 8)$} & \multicolumn{2}{c}{$A_{T}(2 \cdot 2)$} \\
\cline { 2 - 5 }$(1-f)$ & $A_{T E} / A_{T N}$ & Decrease \% & $A_{T E} / A_{T N}$ & Decrease \% \\
\hline 0.60 & 0.78 & 22 & 0.85 & 15 \\
0.30 & 0.65 & 35 & 0.69 & 31 \\
0.23 & 0.55 & 45 & 0.56 & 44 \\
& 0.48 & 52 & 0.40 & 60 \\
\hline
\end{tabular}

*Delayed effect 18 min after maximum phase.

disc from 0.60 to 0.30 , or by a factor of $2, A_{T}(1.8)$ dropped from 0.78 to 0.65 , or by a factor of $1 \cdot 2$ and similarly $A_{T}(2 \cdot 2)$ fell by a factor $1 \cdot 23$, but not by a factor $(1-f)^{1 / 2}$ or $(2)^{1 / 2}$ in this case. Further, for $23 \%$ open disc at maximum phase, $A_{T}(1.8)$ fell to 0.55 and $A_{T}(2.2)$ to 0.56 of normal. This means that for a further decrease in the visible part of the solar disc by a factor of 1.3, the change in $A_{T}$ was faster than for the earlier part of the eclipse. After the delayed minimum, $A_{T}(1 \cdot 8)$ almost half of normal, it recovered quickly to the normal value a little before the last contact within $\pm 15 \mathrm{~dB} \mathrm{MHz}^{2}$ or absorption within $\pm 2 \mathrm{~dB}$ limits. It should be pointed out that the recovery of $A_{T}(2 \cdot 2)$ was faster 
than that of $A_{T}(1 \cdot 8)$. Thus, in contrast to the symmetry seen in the optical eclipse before and after the maximum phase, the changes in total absorption $L_{t}$ as well as in $A_{T}(f)$ showed considerable asymmetry.

\section{Discussion and conclusions}

The decrease in the total ionospheric absorption $L_{t}$ of about $41 \%$ on $1.8 \mathrm{MHz}$ and $46 \%$ on $2.2 \mathrm{MHz}$, and the corresponding decreases of $52 \%$ in $A_{T}(1 \cdot 8)$ and $60 \%$ in $A_{T}(2 \cdot 2)$ from the normal value due to the eclipse following its maximum phase do not seem to be in conformity with the area of the solar disc left unobscured, nor is the rate of decrease in $L_{t}$ or $A_{T}(f)$ in tune with the instantaneous changes in the obscuration of the solar disc. In the E-region, the maximum fall in $N m E$ on the eclipse-day was $45 \%$ of the normal. Let us examine how these results compare with known facts. The active regions on the sun emitting UV/EUV radiations responsible for ionization in the $D$ and $E$ regions are not uniformly distributed over the whole solar disc (Friedman 1959; Hall 1970, NOAA 1980). The small scatter seen in the changes of $L_{t}$ and $A_{T}(f)$ in the initial stage of the eclipse could be a real effect indicating obscuration of irregularly distributed active areas or the so-called 'hot-spots' which on the average are smoothed out to normal. Secondly, the decrease in electron density $N(h)$ is not necessarily in direct proportion to the decrease in electron-ion production rate $q$ or the ionizing radiation flux $F$ at different altitudes, e.g. $N$ varies as $q$ in the lower $D$-region whereas $N$ may vary as $(q)^{1 / 2}$ or in some other way in the upper $D$ and lower $E$ regions (Jespersen and Pedersen 1970; Haug et al 1970; Kane 1970). Such a dependence of $N$ on $q$ would cause comparatively greater decrease in $L_{n d}$ in the $D$-region than increase in $L_{d}$ in the $E$-region where height of reflection increases at a given frequency during the eclipse. Elwert (1958) and Taubenheim and Serafimov (1969) showed that 10 to $15 \%$ of coronal $x$-rays remained unobscured even during totality of the solar eclipse, but according to Willmore (1970) and Haug et al (1970), the quiet-sun x-rays are not important for ionization at around $75 \mathrm{~km}$ altitude. All these factors concerning radiation and ionization suggest a greater decrease in $L_{n d}$ than an increase in $L_{d}$. Hence, neither $L_{t}$ nor $A_{T}(f)$ is likely to have any definite correlation with the unobscured part of the solar disc.

To substantiate the above conclusion, $L_{n d}$ was found from a rough estimate of $L_{d}$ by using Jaeger's (1947) method. The results obtained at the time corresponding to the maximum fall in foE are given in table 2, which shows a clear decrease in $L_{n d}$ and an increase in $L_{d}$ on the eclipse-day as compared to their normal values, the effect being

Table 2. Content of $L_{\mathrm{m}}$ and $L_{d}$ in total absorption on the eclipse day and control days at the time corresponding to maximum drop in foE during the eclipse.

\begin{tabular}{|c|c|c|c|c|}
\hline \multirow{2}{*}{$\stackrel{f}{f(\mathrm{MHz})}$} & \multicolumn{2}{|c|}{$\begin{array}{l}\text { Control days } \\
\text { normal }\end{array}$} & \multicolumn{2}{|c|}{ Eclipse-day } \\
\hline & $L_{n d}$ & $\begin{array}{c}L_{d} \\
\text { in } \% \text { of } L_{t N}\end{array}$ & $L_{*}$ & $\begin{array}{c}L_{d} \\
\text { in } \% \text { of } L_{t E}\end{array}$ \\
\hline 1.8 & 82 & 18 & 68 & 32 \\
\hline 2.2 & 75 & 25 & 47 & 53 \\
\hline
\end{tabular}


larger on $2.2 \mathrm{MHz}$ than on $1.8 \mathrm{MHz}$. If, however, only the magnitudes in $\mathrm{dB}$ of the $L_{n d}$ component on the normal and the eclipse days are considered, a decrease of $51 \%$ was found in it on $1.8 \mathrm{MHz}$ and $65 \%$ on $2.2 \mathrm{MHz}$ during maximum drop, mainly due to decrease in the $D$-region ionization. Incidentally, it is worth noting that the content of deviative component $L_{d}$ was $53 \%$ in the total absorption observed on the eclipse-day at $2.2 \mathrm{MHz}$ when $f o E$ dropped to $2.7 \mathrm{MHz}$. Thus, interestingly, the drop in $A_{T}(f)$ is greater than that in $L_{4}$ by about $11-14 \%$, but the remarkable point is that the drop (52\%) in $A_{T}$ on the lower frequency $1.8 \mathrm{MHz}$ is very nearly the same as that $(51 \%)$ in $L_{n d}$ on the eclipse-day. The rapid decrease and recovery of $A_{T}(f)$ on $2.2 \mathrm{MHz}$ is probably due to faster change in ionization in the upper $D$-region by the covering and uncovering of the solar ionizing radiations.

Rocket flights were made at SHAR $\left(13^{\circ} \mathrm{N}, 80^{\circ} \mathrm{E}\right)$ and Thumba $\left(8^{\circ} \mathrm{N}, 76^{\circ} \mathrm{E}\right)$ only on the eclipse-day of which one of the two flights at the former and three out of four at the latter were successful (Gupta and Chakravarty 1982). From the rocket-borne probe measurements, it was found that the electron density decreased significantly at all levels in the $D$ and $E$ regions as the eclipse progressed which also included the normal diurnal variation, but it was not possible to find how much was the decrease relative to that on the normal or control days. Acharya et al (1982) constructed $N(h)$ profiles at Udaipur $\left(24.6^{\circ} \mathrm{N}, 73.6^{\circ} \mathrm{E}\right)$ so as to match the observed $L_{t}$ on the control days and the eclipse-day at the time corresponding to the maximum phase of the eclipse $(72 \%, 1547 \mathrm{hr}$ IST). They showed a decrease in $N(h)$ by a factor of about 7 at $70 \mathrm{~km}$ altitude, but hardly by a factor of 1.5 at $80-85 \mathrm{~km}$. From our experience in constructing model $N(h)$ profiles using ground-based measurements of ionospheric absorption, partial reflections and ionosonde data (Gupta and Kotadia 1979; Datta et al 1983) and the method adopted by Mechtly and Bilitza (1974) to fit rocket-borne measurements which is also incorporated in the international reference ionosphere (IRI) modelling, we constructed $N(h)$ profiles at Ahmedabad for different solar zenith angles on the normal and the eclipse days such that the calculated and measured $L_{t}$ were in agreement. $L_{t}$ from $70 \mathrm{~km}$ altitude upto the height of reflection of the radio waves at the two frequencies was calculated by the generalized magnetoionic theory of Sen and Wyller (1960). These $N(h)$ profiles are not presented here. Instead, decreases in $N(h)$ on the eclipse-day from its control days mean values at different altitudes are shown in figure 4 for the time corresponding to the

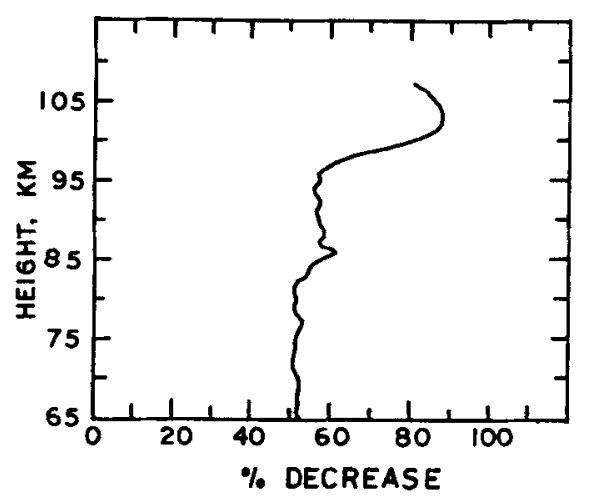

Figure 4. Percentage decrease of electron density on the eclipse-day from that of the control days' mean value at different heights. 
lowest value of $A_{T}$ to show clearly the direct connection of decrease in radiowave absorption with that in $N(h)$. It is seen that the $D$-region electron density in the height range $70-90 \mathrm{~km}$ decreased by $52-56 \%$ i.e. approximately by a factor of 2 and it is about the same as the decrease in $A_{T}(f)$ and also in $L_{n d}$, and this agreement seems to be better at the lower frequency. These results amply prove the reliability of our method for constructing $N(h)$ profile. Another thing to note is that there was about $90 \%$ decrease in electron density near $105 \mathrm{~km}$ altitude, though the decrease in $\mathrm{NmE}$ was only $45 \%$ of the normal value. This should be distinguished from the increase in $h m E$ on the eclipseday by about $10 \mathrm{~km}$ over the normal value of $110 \mathrm{~km}$ with the result that $N$ decreased sharply at around $105 \mathrm{~km}$. The increase in height of reflection and decrease in $N \mathrm{mE}$ result in the increase of deviative absorption $L_{d}$ as shown before, more so at the frequency closer to foE.

Finally, the time-delay of $18 \mathrm{~min}$ in the absorption or $A_{T}(f)$ and $f o E$ minima after the maximum phase of the eclipse is just half of what is normally found in February for their diurnal maximum after noontime. This result is in conformity with the theoretical considerations (Rydbeck 1956) and the delay reported by Davies (1956) for an eclipse in the forenoon of summer at a place in auroral zone. But, it may vary from 0 to 23 minutes as reported in literature depending on the latitude of the place, the season and the time of maximum phase of the eclipse. The initial slow rate of decrease in $A_{T}(f)$ after the first contact may be due to obscuration of only a small amount of ionizing radiation flux coming from high latitudes of the solar disc, and in addition, to some effect of ionospheric sluggishness to the causal phenomena.

\section{Acknowledgements}

The authors wish to express their grateful thanks to the Council of Scientific and Industrial Research, and to the University Grants Commission for financial support.

\section{References}

Acharya B L, Yagnik H K, Bansal T C, Vijayvargia S K and Rai R K 1982 Proc. Indian Natl. Sci. Acad. A48 Suppl. 3388

Bischoff K and Taubenheim J 1967 J. Atmos. Terr. Phys. 291063

Chandra H, Sethia G, Vyas G D, Deshpande M R and Vats H O 1981 Indian J. Rad. Space Phys. 1045

Datta G, Pradhan S N and Kotadia K M 1983 Adv. Space Res. 10209

Davies K 1956 Solar eclipses and the ionosphere (eds) W J G Beynon and G M Brown (London: Pergamon Press) p. 40

Elwert G 1958 J. Atmos. Terr. Phys. 12187

Friedman H 1959 J. Geophys. Res. 641751

George P L 1971 J. Atmos. Terr. Phys. 331993

Gupta S P and Chakravarty D K 1982 Proc. Indian Natl. Sci. Acad. A48 Suppl. 3518

Gupta A and Kotadia K M 1979 J. Geophys. (FRG) 4623

Hall J E 1970 J. Atmos. Terr. Phys. 321849

Haug A, Thrane E V, Tsagakis E and Anastassiades M 1970 J. Atmos. Terr. Phys. 321865

Jaeger J C 1947 Proc. Phys. Soc. (London) 5987

Jani K G, Datta G, Patel D B and Kotadia K M 1982 Proc. Indian Natl. Sci. Acad. A48 Suppl. 3316

Jespersen M and Pedersen M B 1970 J. Atmos. Terr. Phys. 321859

Kane J A 1970 Solar eclipses and the ionosphere (ed.) M Anastassiades (New York: Plenum Press) p. 199

Kotadia K M, Chhipa G M and Taubenheim J 1977 Indian J. Radio Space Phys. 61 
Landini N, Russo D and Tagliaferri G L 1966 Nature (London) 211393

Leid F 1956 Solar eclipses and the ionosphere (ed.) W J G Beynon and G M Brown (London: Pergamon Press) p. 21

Mechtly E A and Bilitza D 1974 Models of D-region electron concentrations, Rep. IPW-WB1, Inst. Phys. Weltraumforschung, Freiburg, FRG

Piggott W R 1956 Solar eclipses and the ionosphere (eds) W J G Beynon and G M Brown (London: Pergamon Press) p. 106

Rawer $\mathrm{K} 1956$ Solar eclipses and the ionosphere (ed.) W J G Beynon and G M Brown (London: Pergamon Press) p. 102

Rydbeck O E H 1956 Solar eclipses and the ionosphere (eds) W J G Beynon and G M Brown (London: Pergamon Press) p. 14

Samuel J C and Bradley P A 1975 J. Atmos. Terr. Phys. 37131

Sen H K and Wyller A A 1960 J. Geophys. Res. 653931

Sechrist C F Jr 1970 Radio Sci. 5663

NOAA 1980 Solar geophysical data, NOAA, Boulder, U.S.A. (monthly bulletins)

Taubenheim J and Serafimov K 1969 J. Atmos. Terr. Phys. 31307

Willmore A P 1970 J. Atmos. Terr. Phys. 321855 